\title{
METHODS
}

\section{Chip-platform fabrication:}

Platform design and fabrication schematics can be found in Supplementary Fig. 6. Microtextured surfaces for cell migration studies were fabricated using a polydimethylsiloxane (PDMS) (Sylgard) replica molding process ${ }^{1}$. Briefly, a 10:1 mixture of PDMS:curing agent was cast on the surface of a photolithographically-patterned silicon ( $\mathrm{Si}$ ) wafer. The PDMS was allowed to cure at room temperature for $>48 \mathrm{~h}$ before demolding.

Two-dimensional PDMS-based nanoelectroporation (NEP) devices were also molded from a photolithographically-patterned Si master ${ }^{2,3}$. Such masters were fabricated using a combination of projection and contact lithography. Briefly, SPR950 nanowires ( 200 nm) were patterned by projection lithography (GCA 6100C Stepper) on a Si wafer. This pattern was then transferred onto the Si surface by reactive ion etching using SF6 (LAM Autoetch 490 Poly/Nitride Plasma Etcher). SU-8 microridges were then patterned/aligned on the Si nanowires. Subsequently, the NEP devices were fabricated by casting a layer of hard PDMS (1:1 PDMS:curing agent) on the Si masters followed by a softer layer (10:1 PDMS:curing agent). After demolding, the PDMS was baked further at $170^{\circ} \mathrm{C}$ for $>15 \mathrm{~h}$. Finally, the textured PDMS surface and NEP device were integrated into a single chip-platform via oxygen plasma bonding.

Si-based 3D NEP devices for large-scale single-clone interrogation were fabricated as described previously ${ }^{4}$. Briefly, $\sim 1 \mathrm{~cm}^{2}$ NEP devices were fabricated from thinned $(\sim 200 \mu \mathrm{m})$ double-side polished (100) silicon wafers. A $\sim 1.5 \mu \mathrm{m}$ thick layer of photoresist was spin coated on the silicon surface and nanoscale channels $(\sim 10 \mu \mathrm{m}$ deep) were etched into the Si substrate using projection lithography and deep reactive ion etching. Microscale reservoirs were then defined on the back 
side of the Si substrate using contact lithography and DRIE. Finally, a 100-200 nm-thick insulating/protective layer of silicon nitride was deposited on the surface via PECVD.

\section{Cell culture and analysis:}

GBM samples were collected at The Ohio State University in accordance with an institutional review board-approved protocol. Freshly resected tumor samples were dissociated and established GSCs were cultured in DMEM/F12 (Invitrogen) supplemented with B27 (Miltenyi Biotech), heparin $(2.5 \mu \mathrm{g} / \mathrm{ml})$, bFGF (Peprotech, $20 \mathrm{ng} / \mathrm{ml})$, and EGF (Peprotech, $20 \mathrm{ng} / \mathrm{ml}$ ). bFGF and EGF were added twice a week. The phenotypes of these neurospheres were molecularly characterized as previously described ${ }^{5,6}$. GSE67089 datasets in the NCBI Gene Expression Omnibus (GEO) were analyzed to compare expression profiles for migration-related genes between GBM157 and GBM528. The data were log transformed, median centered, and processed with Java Treview software.

Cell migration studies were conducted on an inverted microscope fitted with a cell culture chamber. GSC neurospheres were dissociated into monodispersed cell suspensions and then plated on the textured PDMS surface using GSC culture medium without heparin, and supplemented with $10 \%$ fetal bovine serum (FBS). Images were collected every 10 minutes and analyzed using Fiji software as described before ${ }^{1}$.

Nanoelectroporation experiments were conducted as previously described ${ }^{4,7}$. Cells were loaded into 2D or 3D NEP devices via optical tweezers or direct surface platting, respectively. For optical tweezers-based loading, the cells were manipulated with a custom-built system using a $3 \mathrm{~W}, 1,064$ $\mathrm{nm}$ laser (Crystal Laser) mounted on an inverted microscope (Olympus IX-71). Cell maneuvering was conducted using power values around $600 \mathrm{~mW}$. Direct surface platting, on the other hand, was 
carried out simply by letting the cells adhere on the nanochanneled surface of the 3D NEP component using GSC media (without heparin) supplemented with 10\% FBS. The cargo to be transfected (e.g., molecular beacons, anti-miR) was loaded in phosphate buffered saline. A pulsed electric field was then applied across the nanochannels using a Biorad Gene Pulser Xcell power supply. The applied voltage ranged between $200-250 \mathrm{~V}$, and the pulse length varied between 5-20 ms. Anti-miR363 was purchased from ABI. Temozolomide and molecular beacons (Vimentin, CD44, GAPDH, CD133, MLK4, and miR363) were purchased from Sigma-Aldrich. Molecular beacons are stem-and-loop structured oligodeoxynucleotides with a fluorophore on one end and a quencher at the other ${ }^{8,9}$. Once the beacon enters the cell and finds a complementary target, hybridization would occur, which causes the quencher and probe to separate, consequently, this results in a fluorescence signal that allows for in situ detection of specific targets (e.g., mRNAs, oligo RNAs) inside living cells.

\section{Statistical Analysis:}

All experiments were performed in triplicate. All statistics were performed in SigmaPlot version 13.0.

\section{REFERENCES}

1. $\quad$ Gallego-Perez, D.; Higuita-Castro, N.; Denning, L.; DeJesus, J.; Dahl, K.; Sarkar, A.; Hansford, D. J. Lab Chip 2012, 12, (21), 4424-32.

2. Zhao, X.; Wu, Y.; Gallego-Perez, D.; Kwak, K. J.; Gupta, C.; Ouyang, X.; Lee, L. J. Anal Chem 2015, 87, (6), 3208-15.

3. Gupta, C.; Liao, W. C.; Gallego-Perez, D.; Castro, C. E.; Lee, L. J. Biomicrofluidics 2014, 8, (2), 024114.

4. $\quad$ Chang, L.; Gallego-Perez, D.; Zhao, X.; Bertani, P.; Yang, Z.; Chiang, C. L.; Malkoc, V.; Shi, J.; Sen, C. K.; Odonnell, L.; Yu, J.; Lu, W.; Lee, L. J. Lab Chip 2015, 15, (15), 3147-53. 5. $\quad$ Mao, P.; Joshi, K.; Li, J.; Kim, S. H.; Li, P.; Santana-Santos, L.; Luthra, S.; Chandran, U. R.; Benos, P. V.; Smith, L.; Wang, M.; Hu, B.; Cheng, S. Y.; Sobol, R. W.; Nakano, I. Proc Natl Acad Sci U S A 2013, 110, (21), 8644-9. 
6. Guvenc, H.; Pavlyukov, M. S.; Joshi, K.; Kurt, H.; Banasavadi-Siddegowda, Y. K.; Mao, P.; Hong, C.; Yamada, R.; Kwon, C. H.; Bhasin, D.; Chettiar, S.; Kitange, G.; Park, I. H.;

Sarkaria, J. N.; Li, C.; Shakhparonov, M. I.; Nakano, I. Clin Cancer Res 2013, 19, (3), 631-42.

7. Boukany, P. E.; Morss, A.; Liao, W. C.; Henslee, B.; Jung, H.; Zhang, X.; Yu, B.; Wang, X.; Wu, Y.; Li, L.; Gao, K.; Hu, X.; Zhao, X.; Hemminger, O.; Lu, W.; Lafyatis, G. P.; Lee, L. J. Nat Nanotechnol 2011, 6, (11), 747-54.

8. $\quad$ Santangelo, P.; Nitin, N.; Bao, G. Ann Biomed Eng 2006, 34, (1), 39-50.

9. Sokol, D. L.; Zhang, X.; Lu, P.; Gewirtz, A. M. Proc Natl Acad Sci U S A 1998, 95, (20), 11538-43. 